\title{
Absence of high-energy spectral concentration for Dirac systems with divergent potentials
}

\author{
M. S. P. Eastham \\ Department of Computer Science, Cardiff University, \\ PO Box 916, Cardiff CF24 3XF, UK \\ K. M. Schmidt \\ School of Mathematics, Cardiff University, \\ Cardiff CF24 4AG, UK (schmidtkm@cardiff.ac.uk)
}

(MS received 14 May 2004; accepted 13 January 2005)

\begin{abstract}
It is known that one-dimensional Dirac systems with potentials $q$ which tend to $-\infty$ (or $\infty$ ) at infinity, such that $1 / q$ is of bounded variation, have a purely absolutely continuous spectrum covering the whole real line. We show that, for the system on a half-line, there are no local maxima of the spectral density (points of spectral concentration) above some value of the spectral parameter if $q$ satisfies certain additional regularity conditions. These conditions admit thrice-differentiable potentials of power or exponential growth. The eventual sign of the derivative of the spectral density depends on the boundary condition imposed at the regular end-point.
\end{abstract}

\section{Introduction}

By the classical results of Titchmarsh (see $[23, \S 5.7]$ and $[24, \S 5.10]$ ) and Hartman [14], the spectrum of a one-dimensional Schrödinger operator

$$
l:=-\frac{\mathrm{d}^{2}}{\mathrm{~d} x^{2}}+q(x) \quad(0 \leqslant x<\infty)
$$

fills the whole real line if $\lim _{x \rightarrow \infty} q(x)=-\infty$ subject to the growth condition

$$
q(x)=O\left(x^{2}\right) \quad(x \rightarrow \infty)
$$

and certain further restrictions. Thus, for example, Titchmarsh imposes conditions on $q^{\prime}$ and $q^{\prime \prime}$, while Hartman requires $q$ to be decreasing with (1.2) tightened to $o\left(x^{2}\right)$ (see also [5], [12, §33] and [17]). An example by Halvorsen [13] shows that if only (1.2) is imposed, the spectrum may have gaps and eigenvalues. On the other hand, in the presence of suitable regularity, Titchmarsh [23, 24] and Hinton [15] showed that the spectral measure $\varrho$ is absolutely continuous on the whole real line (see also $[22,25])$.

However, this absolute continuity does not necessarily mean that the spectrum is completely homogeneous; indeed the spectral density $\varrho^{\prime}$ may vary and in particular have local maxima, which are known as points of spectral concentration. Such points have been observed computationally to be associated with eigenvalues of an unperturbed problem which disappear after addition of a perturbation tending to $-\infty$, 
as in the Stark effect, for example [2]. Under certain conditions on the potential, it is known that the set of spectral concentration points is bounded, that is, the spectral density is monotonic for large values of the spectral parameter [9].

In this paper, we consider the relativistic analogue of (1.1), the Dirac operator

$$
h:=-\mathrm{i} \sigma_{2} \frac{\mathrm{d}}{\mathrm{d} x}+\sigma_{3}+q(x) I_{2},
$$

where

$$
\sigma_{2}=\left(\begin{array}{cc}
0 & -\mathrm{i} \\
\mathrm{i} & 0
\end{array}\right) \quad \text { and } \quad \sigma_{3}=\left(\begin{array}{cc}
1 & 0 \\
0 & -1
\end{array}\right)
$$

are Pauli matrices,

$$
I_{2}=\left(\begin{array}{ll}
1 & 0 \\
0 & 1
\end{array}\right)
$$

is the unit matrix, and $q \in L_{\text {loc }}^{1}(0, \infty)$ satisfies

$$
\lim _{x \rightarrow \infty} q(x)=-\infty .
$$

At $x=0$ we impose a boundary condition

$$
y_{1}(0) \cos \alpha-y_{2}(0) \sin \alpha=0,
$$

with fixed $\alpha \in \mathbb{R}$. At infinity, the Dirac system is in the limit-point case. Hence, the operator $h$ with boundary condition (1.5) has a unique self-adjoint realization in the Hilbert space $L^{2}(0, \infty)^{2}$. Its domain is the space of locally absolutely continuous functions $y:[0, \infty) \rightarrow \mathbb{C}^{2}$ satisfying (1.5) and such that $y, h y \in L^{2}(0, \infty)^{2}$.

Although the Dirac operator has qualitatively similar spectral properties to the Schrödinger operator, there are also some fundamental differences. For example, the main part of (1.3) is unitarily equivalent to its negative, so the operators with potentials $q$ and $-q$ have the same spectra, only with reversed sign of the spectral parameter. Thus, the condition (1.4), but with $+\infty$ in place of $-\infty$, gives a Dirac operator with identical qualitative spectral properties but, in contrast, (1.1) then has a purely discrete spectrum.

It was shown by Erdélyi [10] that the spectrum of (1.3), with (1.4) imposed, is purely absolutely continuous and covers the whole real line if

$$
\int^{\infty} \frac{\left|q^{\prime}\right|}{q^{2}}<\infty
$$

for absolutely continuous $q$. More recently, it was shown by Schmidt [19] that (1.6) can be weakened to the condition that $1 / q$ be a function of bounded variation. This includes eventually monotonic though not necessarily continuous $q$, thus vindicating a conjecture of Rose and Newton [18]. This type of criterion also extends to spherically symmetric three-dimensional Dirac operators, even with a variable mass term, provided the latter is dominated by the potential [21]. On the other hand, (1.4) alone, without a condition of type (1.6), is not sufficient for the spectrum to fill the real line $[20]$.

In view of these results, it seems a natural question to ask whether the spectral density of such Dirac operators with purely absolutely continuous spectra can have 
local maxima. Our main result (theorem 4.1) shows that there are no points of spectral concentration beyond some value of the spectral parameter provided that $q$ satisfies certain regularity conditions in addition to (1.4) and (1.6). In particular, we include thrice-differentiable potentials of power or exponential growth. As a preparatory step for the proof, we develop an integral formula for the spectral density $\varrho^{\prime}$ in $\S 2$, involving the Prüfer angle of a solution of the eigenvalue equation (theorem 2.3). Along the way, we also find an asymptotic estimate for the derivative of the Prüfer angle with respect to the spectral parameter (theorem 2.2). In $\S 3$, we introduce the detailed conditions on $q$ which we require to show that $\varrho^{\prime \prime}$ is ultimately of one sign, which turns out to depend on the boundary condition at 0 . Section 4 contains the main result and its proof for boundary conditions with $2 \alpha \neq \pi / 2 \bmod \pi$. The exceptional values $2 \alpha=\pi / 2 \bmod \pi$ require slightly stronger regularity of potentials of exponential growth and are dealt with in section 5, leading to an analogous result (theorem 5.1).

\section{Asymptotics of solutions and the spectral density}

In the case of the Schrödinger operator (1.1) considered in, for example, $[1,3,7,8$, $11,24, \S \S 5.7$ and 5.10], an integral formula for the spectral density is derived as a consequence of the asymptotic form of the solutions of the equation $l y=\lambda y$ as $x \rightarrow \infty$ (see [6]). Here $\lambda$ is the complex spectral parameter. A similar approach could be applied to the Dirac operator (1.3); however, we follow a different strategy here, motivated by the method used in [19] to prove the boundedness of the solutions of the corresponding eigenvalue equation with a real spectral parameter; it will in fact not be necessary to study the complex eigenvalue equation.

Throughout this section, we assume that $q$ satisfies the conditions (1.4) and (1.6). By (1.4) we can assume that $q$ is bounded from above on $[0, \infty)$. Furthermore, since we are concerned with $\mu$ in an interval $\left[\mu_{0}, \infty\right)$, we can assume that $\mu_{0}$ is already so large that

$$
Q(x, \mu):=\mu-q(x)>1 \quad\left(0 \leqslant x<\infty, \mu_{0} \leqslant \mu<\infty\right) .
$$

In order to obtain the asymptotics of the solutions of the eigenvalue equation

$$
h y=\mu y
$$

with $\mu \in \mathbb{R}$, it proves convenient to introduce the Prüfer transformation

$$
y_{1}(x, \mu)=u(x, \mu) \sin \vartheta(x, \mu), \quad y_{2}(x, \mu)=u(x, \mu) \cos \vartheta(x, \mu),
$$

where

$$
y=\left(\begin{array}{l}
y_{1} \\
y_{2}
\end{array}\right) \quad \text { and } \quad u=\sqrt{y_{1}^{2}+y_{2}^{2}}>0 .
$$

The eigenvalue equation (2.2) is then equivalent to the pair of differential equations

$$
\vartheta^{\prime}=\mu-q+\cos 2 \vartheta
$$

and

$$
(\log u)^{\prime}=\sin 2 \vartheta
$$


The second equation gives

$$
u(t, \mu)=u(0, \mu) \exp \left(\int_{0}^{t} \sin 2 \vartheta(x, \mu) \mathrm{d} x\right)
$$

directly. The boundary condition (1.5) leads to the initial condition $\vartheta(0, \mu)=\alpha$.

Theorem 2.1. Let $q$ satisfy (1.4) and (1.6), and let $\vartheta, u$ be Prüfer variables of a solution of (2.2) with initial values

$$
\vartheta(0, \mu)=\alpha, \quad u(0, \mu)=1 .
$$

Then

(i) there are positive constants $C$ and $c$ such that $c \leqslant u(x, \mu) \leqslant C$ on $[0, \infty) \times$ $\left[\mu_{0}, \infty\right)$;

(ii) $u(x, \mu)=1+o(1)(\mu \rightarrow \infty)$, where the o(1) term is uniform in $x$;

(iii) $u(\infty, \mu):=\lim _{x \rightarrow \infty} u(x, \mu)$ exists and $u(\infty, \mu)=1+o(1)(\mu \rightarrow \infty)$.

Proof. Observing that

$$
\left(\log \frac{q-\mu-\cos 2 \vartheta}{q-\mu}\right)^{\prime}=\frac{q^{\prime} \cos 2 \vartheta}{(q-\mu)(q-\mu-\cos 2 \vartheta)}-2 \sin 2 \vartheta
$$

we find, using (2.5), that

$$
\begin{aligned}
2 \lim _{x \rightarrow \infty} \log u(x, \mu) & =\int_{0}^{\infty} 2 \sin 2 \vartheta \\
& =\log \frac{q(0)-\mu-\cos 2 \alpha}{q(0)-\mu}+\int_{0}^{\infty} \frac{q^{\prime} \cos 2 \vartheta}{(q-\mu)(q-\mu-\cos 2 \vartheta)} ;
\end{aligned}
$$

the integral, and hence the limit in (iii), exists by virtue of (1.6) and (2.1). It is clear that both the logarithm and the integral are bounded for $\mu \in\left[\mu_{0}, \infty\right)$; the former is $O(1 / \mu)(\mu \rightarrow \infty)$, whereas the latter is bounded above by

$$
\int_{0}^{\infty}\left|q^{\prime}\right|\left(\mu_{0}-q-1\right)^{-2}
$$

and tends to 0 as $\mu \rightarrow \infty$ by Lebesgue's dominated convergence theorem.

For later use, we observe that theorem 2.1 also yields an asymptotic estimate for the derivative of the Prüfer angle $\vartheta$ with respect to the spectral parameter.

THEOREM 2.2. Under the hypotheses of theorem 2.1,

$$
\frac{\partial \vartheta}{\partial \mu}(x, \mu)=x(1+o(1)) \quad(\mu \rightarrow \infty),
$$

where the o-term is uniform in $x \in[0, \infty)$. 
Proof. Together with (2.4), differentiation of (2.3) with respect to $\mu$ gives

$$
\left(\frac{\partial \vartheta}{\partial \mu}\right)^{\prime}=1-2(\log u)^{\prime} \frac{\partial \vartheta}{\partial \mu}
$$

from which it follows that

$$
\frac{\partial \vartheta}{\partial \mu}(x, \mu)=\frac{1}{u^{2}(x, \mu)} \int_{0}^{x} u^{2}(t, \mu) \mathrm{d} t
$$

the two sides agreeing at $x=0$ because of (2.6). Hence,

$$
\frac{1}{x}\left(x-\frac{\partial \vartheta}{\partial \mu}(x, \mu)\right)=\frac{1}{x} \int_{0}^{x}\left(1-\frac{u^{2}(t, \mu)}{u^{2}(x, \mu)}\right) \mathrm{d} t \rightarrow 0 \quad(\mu \rightarrow \infty)
$$

uniformly in $x$, by theorem 2.1 (ii).

We now proceed to derive the following convenient formula for the spectral density.

THEOREM 2.3. Let $q$ satisfy (1.4) and (1.6), and let $\vartheta, u$ be the Prüfer variables for a solution of (2.2) with the initial values (2.6). Then the spectral density of $h$ satisfies

$$
\pi \varrho_{\alpha}^{\prime}(\mu)=\frac{1}{u^{2}(\infty, \mu)}=\exp \left(-2 \int_{0}^{\infty} \sin 2 \vartheta(x, \mu) \mathrm{d} x\right) .
$$

Proof. We use the definition of the spectral function $\varrho_{\alpha}$ as a limit of the spectral functions of regular problems on a finite interval. For $b \in(0, \infty)$, the boundary-value problem for $h$ on $(0, b)$ with the boundary condition

$$
y(b) \cdot\left(\begin{array}{c}
\cos \beta \\
-\sin \beta
\end{array}\right)=0
$$

has the spectral (step) function

$$
\varrho_{\alpha \beta}(b, \mu)=\sum_{\lambda \text { e.v. }} \frac{1}{\int_{0}^{b} u^{2}(x, \lambda) \mathrm{d} x},
$$

where the sum extends over all eigenvalues $\lambda$ of this restricted boundary-value problem which lie in the interval $\left(\mu_{0}, \mu\right]$ (see $[16$, ch. $\left.3, \S 1]\right)$.

For a given $b$, we denote these eigenvalues by $\lambda_{K}(\beta), \lambda_{K+1}(\beta), \ldots, \lambda_{L}(\beta)$ and we consider $\beta$ to be varying between 0 and $\pi$. The Prüfer angle at $b$ is a monotonically increasing function of the spectral parameter (see, for example, (2.8)) and, in view of the boundary condition at $b$,

$$
\vartheta\left(b, \lambda_{r}(\beta)\right)=\beta+r \pi
$$

holds. Hence $\lambda_{r}(\beta)$ varies monotonically between $\lambda_{r-1}(\pi)$ and $\lambda_{r}(\pi)$ as $\beta$ varies between 0 and $\pi$. Therefore, integration of (2.10) with respect to $\beta$ gives

$$
\begin{aligned}
\int_{0}^{\pi} \varrho_{\alpha \beta}(b, \mu) \mathrm{d} \beta= & \int_{\lambda_{K}^{-1}\left(\mu_{0}\right)}^{\pi} \frac{1}{\int_{0}^{b} u^{2}\left(x, \lambda_{K}(\beta)\right) \mathrm{d} x} \mathrm{~d} \beta+\int_{0}^{\pi} \frac{1}{\int_{0}^{b} u^{2}\left(x, \lambda_{K+1}(\beta)\right) \mathrm{d} x} \mathrm{~d} \beta \\
& +\cdots+\int_{0}^{\lambda_{L}^{-1}(\mu)} \frac{1}{\int_{0}^{b} u^{2}\left(x, \lambda_{L}(\beta)\right) \mathrm{d} x} \mathrm{~d} \beta
\end{aligned}
$$


694

$$
\begin{aligned}
& =\int_{\mu_{0}}^{\lambda_{K}(\pi)} \frac{1}{\int_{0}^{b} u^{2}(x, \lambda) \mathrm{d} x} \frac{\partial \vartheta(b, \lambda)}{\partial \lambda} \mathrm{d} \lambda \\
& \quad+\int_{\lambda_{K}(\pi)}^{\lambda_{K+1}(\pi)} \frac{1}{\int_{0}^{b} u^{2}(x, \lambda) \mathrm{d} x} \frac{\partial \vartheta(b, \lambda)}{\partial \lambda} \mathrm{d} \lambda \\
& \quad+\cdots+\int_{\lambda_{L-1}(\pi)}^{\mu} \frac{1}{\int_{0}^{b} u^{2}(x, \lambda) \mathrm{d} x} \frac{\partial \vartheta(b, \lambda)}{\partial \lambda} \mathrm{d} \lambda,
\end{aligned}
$$

by a change of variables. Hence, by (2.8), we obtain

$$
\int_{0}^{\pi} \varrho_{\alpha \beta}(b, \mu) \mathrm{d} \beta=\int_{\mu_{0}}^{\mu} \frac{\mathrm{d} \lambda}{u^{2}(b, \lambda)} .
$$

We now wish to let $b \rightarrow \infty$ under the integral sign on each side of (2.12), and we shall justify this process by dominated convergence. We consider the left-hand side of (2.12) first. As a consequence of theorem 2.1, equation $(2.2)$ has no $L^{2}(0, \infty)$ solutions and hence (1.3) has a purely continuous spectrum and is in the limit-point case at $\infty$; thus

$$
\varrho_{\alpha}(\mu)=\lim _{b \rightarrow \infty} \varrho_{\alpha \beta}(b, \mu)
$$

for all $\mu \geqslant \mu_{0}$ independently of $\beta$ (see [4, ch. 9, theorem 3.1]). Next we observe that $\varrho_{\alpha \beta}(b, \mu)$ is bounded uniformly with respect to both $b$ and $\beta$. Indeed, by (2.11), (2.8) and theorem 2.1 (i), we have

$$
\begin{aligned}
(L-K) \pi & =\vartheta\left(b, \lambda_{L}(\beta)\right)-\vartheta\left(b, \lambda_{K}(\beta)\right) \\
& =\int_{\lambda_{K}(\beta)}^{\lambda_{L}(\beta)} \frac{\int_{0}^{b} u^{2}(t, \lambda) \mathrm{d} t}{u^{2}(b, \lambda)} \mathrm{d} \lambda \\
& \leqslant\left(\lambda_{L}(\beta)-\lambda_{K}(\beta)\right) \frac{b C^{2}}{c^{2}} \\
& \leqslant\left(\mu-\mu_{0}\right) b \frac{C^{2}}{c^{2}} .
\end{aligned}
$$

Hence, by (2.10),

$$
\varrho_{\alpha \beta}(b, \mu) \leqslant \frac{L-K+1}{b c^{2}} \leqslant \frac{\left(\mu-\mu_{0}\right) C^{2}}{\pi c^{4}}+\frac{1}{b c^{2}} .
$$

Thus, by dominated convergence in (2.13), the left-hand side of (2.12) converges to $\pi \varrho_{\alpha}(\mu)$ as $b \rightarrow \infty$.

On the right-hand side of (2.12), we again apply theorem 2.1 and dominated convergence to obtain finally

$$
\varrho_{\alpha}(\mu)=\frac{1}{\pi} \int_{\mu_{0}}^{\mu} \frac{\mathrm{d} \lambda}{u^{2}(\infty, \lambda)} .
$$

Thus, $\varrho_{\alpha}$ is absolutely continuous with its derivative given by (2.9); the exponential form in (2.9) follows using (2.5) and (2.6). 
The expression (2.9) has obvious similarities with the case of (1.1) considered in $[1,3,7,8]$, but here the conditions on $q$ are quite different. On the face of it, $(2.9)$ presents an additional difficulty for large $\mu$ because, in contrast to the formula used in $[1,3,7,8]$, it does not contain inverse powers of $\mu$. However, in the next two sections we develop a new and efficient method of dealing with (2.9). The aim is to show that $\varrho_{\alpha}^{\prime \prime}(\mu)>0$, implying that the spectral density has no local maximum, for sufficiently large $\mu$. Since, therefore, a $\mu$-derivative of (2.9) is involved, the estimate for $\partial \vartheta / \partial \mu$ in theorem 2.2 will play an essential role.

\section{Conditions on $q$}

We give now some more detailed conditions on $q$ which, in addition to (1.4) and (1.6), lead to estimates as $\mu \rightarrow \infty$ for certain integrals involving $q$ and its first three derivatives. These estimates will be required in our main result in theorem 4.1. We give two sets of conditions, which, apart from a common lower growth estimate for $|q|$, represent power growth of $q$ and exponential growth of $q$, respectively.

Condition $(\mathrm{P})$. For $x$ in some interval $(X, \infty)$, let

$$
|q(x)| \geqslant c x^{a}
$$

and

$$
\left|q^{(k)}(x)\right| \leqslant C x^{a-k} \quad(k \in\{1,2,3\}),
$$

where $a, c$ and $C$ are positive constants.

Condition (E). Let (3.1) hold again and, for some $\delta<1$ and for any $\varepsilon>0$, let

$$
x q^{(k)} /|q|^{1+\delta} \in L(0, \infty) \quad(k \in\{1,2,3\}),
$$

and

$$
q^{\prime}(x) /|q(x)|^{1+\varepsilon}=O(1) \quad(x \rightarrow \infty) .
$$

REMARKS.

(1) Both conditions imply that (1.6) holds. This is obvious in the case of (P). For $(\mathrm{E})$, note that

$$
\frac{q^{\prime}}{q^{2}}=\frac{x q^{\prime}}{q^{1+\delta}} \frac{1}{x q^{1-\delta}}=o\left(\frac{x q^{\prime}}{q^{1+\delta}}\right) \in L(0, \infty),
$$

where we have used $\delta<1$.

(2) Both conditions imply that

$$
\frac{x q^{\prime \prime}}{q^{2}}=o(1), \quad \frac{x q^{\prime}}{q^{2}}=o(1) \quad(x \rightarrow \infty) .
$$

This is again obvious in the case of $(\mathrm{P})$. For $(\mathrm{E})$, we have

$$
\left(\frac{x q^{\prime \prime}}{q^{1+\delta+\varepsilon}}\right)^{\prime}=\frac{x q^{\prime \prime \prime}}{q^{1+\delta+\varepsilon}}+\frac{q^{\prime \prime}}{q^{1+\delta+\varepsilon}}-(1+\delta+\varepsilon) \frac{x q^{\prime} q^{\prime \prime}}{q^{2+\delta+\varepsilon}} .
$$


Each of the three terms on the right is integrable at infinity, and hence $x q^{\prime \prime} / q^{1+\delta+\varepsilon}$ approaches a finite limit as $x \rightarrow \infty$. Choosing $\varepsilon<1-\delta$ gives the first result in (3.2), and the second is proved similarly.

(3) Condition (E) allows both subexponential and superexponential growth of $|q|$, such as $\exp (\sqrt{x})$ and $\exp \left(x^{2}\right)$.

In the following lemma, we give the estimates that we require on the basis of either set of conditions.

Definition 3.1. Let $m, n, s_{1}, s_{2}, s_{3}$ be non-negative integers with $m, n \geqslant 1$. Let $Q$ be as in (2.1), $\vartheta$ a solution of (2.3), and let $f$ be either $\sin 2 n \vartheta$ or $\cos 2 n \vartheta$. Then the integral

$$
I(\mu):=\int_{0}^{\infty} \frac{\left(q^{\prime}\right)^{s_{1}}\left(q^{\prime \prime}\right)^{s_{2}}\left(q^{\prime \prime \prime}\right)^{s_{3}}}{Q^{m}} f
$$

is said to be of type $\left(m ; s_{1}, s_{2}, s_{3}\right)$.

The integrals $I(\mu)$ which arise in $\S \S 4$ and 5 all have $s_{2}, s_{3} \in\{0,1\}$, but part (a) of the following lemma holds in greater generality.

Lemma 3.2. Let $I(\mu)$ be as in (3.3), and define $\sigma:=\sum_{j=1}^{3} s_{j}, \tau:=\sum_{j=1}^{3} j s_{j}$.

(a) Let condition $(P)$ hold, let $m>2$, and let

$$
m-\sigma+\frac{\tau-2}{a}>2 \text {. }
$$

Then

$$
\frac{\mathrm{d} I(\mu)}{\mathrm{d} \mu}=o\left(\mu^{-2}\right) \quad(\mu \rightarrow \infty) .
$$

(b) Let condition (E) hold, and let the pair $\left(s_{2}, s_{3}\right)$ be one of $(0,0),(1,0)$ and $(0,1)$. Also, let

$$
\begin{aligned}
m-\sigma & >2+\delta \quad \text { if } \sigma \neq 0, \\
m>2+\frac{2}{a} & \text { if } \sigma=0 .
\end{aligned}
$$

Then (3.5) again holds.

Proof. The derivative $\mathrm{d} I(\mu) / \mathrm{d} \mu$ is a linear combination of the integrals

$$
\int_{0}^{\infty} \frac{\left(q^{\prime}\right)^{s_{1}}\left(q^{\prime \prime}\right)^{s_{2}}\left(q^{\prime \prime \prime}\right)^{s_{3}}}{Q^{m+1}} f \text { and } \quad \int_{0}^{\infty} \frac{\left(q^{\prime}\right)^{s_{1}}\left(q^{\prime \prime}\right)^{s_{2}}\left(q^{\prime \prime \prime}\right)^{s_{3}}}{Q^{m}} \tilde{f} \frac{\partial \vartheta}{\partial \mu},
$$

where $\tilde{f}$ is also either $\sin 2 n \vartheta$ or $\cos 2 n \vartheta$. Because of theorem 2.2, the first integral in (3.8) is dominated by the second in both cases, and we therefore focus on the latter.

(a) Using theorem 2.2, we can estimate the second integral in (3.8) by a multiple of

$$
\int_{0}^{\infty} \frac{\left(x^{a-1}\right)^{s_{1}}\left(x^{a-2}\right)^{s_{2}}\left(x^{a-3}\right)^{s_{3}} x}{\left(\mu+c x^{a}\right)^{m}} \mathrm{~d} x
$$


which, on splitting the integration at the point $X$ and substituting $x=\mu^{1 / a} t$, becomes

$$
O\left(\mu^{-m}\right)+\mu^{-m+\sigma-(\tau-2) / a} \int_{\mu^{-1 / a} X}^{\infty} \frac{t^{a \sigma-\tau+1}}{\left(1+c t^{a}\right)^{m}} \mathrm{~d} t .
$$

This integral converges at $t=\infty$ in view of (3.4), and its value converges or diverges as $\mu \rightarrow \infty$ accordingly as $a \sigma-\tau+2$ is positive or otherwise. In either case, we obtain the estimate $O\left(\mu^{-m}\right)+o\left(\mu^{-2}\right)=o\left(\mu^{-2}\right)$ because of (3.4) and $m>2$.

(b) In the case $\sigma=0$ (and hence $\tau=0$ ), the assertion is proved as in part (a), using assumption (3.1).

Otherwise, we consider first $s_{2}=s_{3}=0$, making $\sigma=s_{1} \geqslant 1$. Then we obtain the estimate of the second integral in (3.8),

$$
\int_{0}^{\infty}\left(\frac{\left|q^{\prime}(x)\right|}{Q(x, \mu)^{1+\varepsilon}}\right)^{\sigma-1} \frac{x\left|q^{\prime}(x)\right|}{Q(x, \mu)^{1+\delta}} \frac{1}{Q(x, \mu)^{m-(\sigma-1)(1+\varepsilon)-1-\delta}} \mathrm{d} x .
$$

The power of $Q$ in the denominator is positive if $\varepsilon$ is small enough, because $m-$ $(\sigma-1)-1-\delta=m-\sigma-\delta>2$ by (3.6). Now $Q \leqslant \mu+|q|$ by (2.1), and we use both $Q \leqslant \mu$ and $Q \leqslant|q|$ to find that (3.9) is estimated by

$$
\mu^{-m+(\sigma-1)(1+\varepsilon)+1+\delta} \int_{0}^{\infty}\left(\frac{\left|q^{\prime}(x)\right|}{|q(x)|^{1+\varepsilon}}\right)^{\sigma-1} \frac{x\left|q^{\prime}(x)\right|}{|q(x)|^{1+\delta}} \mathrm{d} x .
$$

The integral here exists by the assumptions of condition (E), and the estimate $o\left(\mu^{-2}\right)$ follows from (3.6), again by suitable choice of $\varepsilon$.

In the cases $s_{2}=1, s_{3}=0$ and $s_{2}=0, s_{3}=1$, we have $\sigma=s_{1}+1$ and proceed as before, the only difference being that $x\left|q^{\prime}(x)\right|$ in (3.9) is replaced with $x\left|q^{\prime \prime}(x)\right|$ and $x\left|q^{\prime \prime \prime}(x)\right|$, respectively.

\section{Absence of spectral concentration}

THEOREM 4.1. Let $q$ be a potential satisfying (1.4) and (1.6); furthermore, assume that $q$ is twice continuously differentiable and $q^{\prime \prime} \in A C_{\mathrm{loc}}(0, \infty)$, and that either condition $(P)$ or condition (E) holds. Suppose further that

$$
\cos 2 \alpha \neq 0 \text {. }
$$

Then

$$
\pi \varrho_{\alpha}^{\prime \prime}(\mu)=\mu^{-2}(\cos 2 \alpha)(1+o(1)) \quad(\mu \rightarrow \infty) .
$$

In particular, there is an interval $(M, \infty)$ which does not contain any points of spectral concentration.

To prove this theorem, we use (2.9) to consider

$$
\pi \varrho_{\alpha}^{\prime \prime}(\mu)=-2\left(\frac{\mathrm{d}}{\mathrm{d} \mu} \int_{0}^{\infty} \sin 2 \vartheta\right) \exp \left(-2 \int_{0}^{\infty} \sin 2 \vartheta\right) .
$$

However, before differentiating under the integral sign in (4.3), we replace this integral by others which are more obviously convergent. We do this by a process of 
integration by parts, observing that, by (2.3),

$$
1=\frac{\vartheta^{\prime}-\cos 2 \vartheta}{Q} \text {. }
$$

Thus, because of the boundary condition $\vartheta(0, \mu)=\alpha$, we have

$$
\begin{aligned}
\int_{0}^{\infty} \sin 2 \vartheta & =\int_{0}^{\infty} \frac{\vartheta^{\prime}-\cos 2 \vartheta}{Q} \sin 2 \vartheta \\
& =\frac{\cos 2 \alpha}{2 Q(0, \mu)}+\frac{1}{2} \int_{0}^{\infty} \frac{q^{\prime}}{Q^{2}} \cos 2 \vartheta-\frac{1}{2} \int_{0}^{\infty} \frac{1}{Q} \sin 4 \vartheta
\end{aligned}
$$

We then repeat the process with the new integrals arising in this way, until integrals emerge whose derivative with respect to $\mu$ can be estimated by means of lemma 3.2 , thus yielding the $o(1)$ term in (4.2). We formalize this process in the following lemma.

LEMma 4.2. Let $I(\mu)$ be an integral of type $\left(m ; s_{1}, s_{2}, 0\right)$. If

$$
\lim _{x \rightarrow \infty} \frac{\left(q^{\prime}(x)\right)^{s_{1}}\left(q^{\prime \prime}(x)\right)^{s_{2}}}{Q(x, \mu)^{m+1}}=0,
$$

then $I(\mu)$ is a linear combination of the following terms:

(i) integrals of types $\left(m+1 ; s_{1}, s_{2}, 0\right),\left(m+1 ; s_{1}-1, s_{2}+1,0\right)\left(\right.$ if $\left.s_{1} \geqslant 1\right),(m+$ $\left.1 ; s_{1}, s_{2}-1,1\right)\left(\right.$ if $\left.s_{2} \geqslant 1\right)$ and $\left(m+2 ; s_{1}+1, s_{2}, 0\right)$;

(ii) a boundary term $Q^{-(m+1)}(0, \mu)$;

(iii) an integral term

$$
\int_{0}^{\infty} \frac{\left(q^{\prime}(x)\right)^{s_{1}}\left(q^{\prime \prime}(x)\right)^{s_{2}}}{Q(x, \mu)^{m+1}} \mathrm{~d} x .
$$

Proof. Indeed, applying the same method as in (4.4), we find that

$$
\begin{aligned}
I(\mu)= & \int_{0}^{\infty} \frac{\left(q^{\prime}\right)^{s_{1}}\left(q^{\prime \prime}\right)^{s_{2}}}{Q^{m+1}}\left(\vartheta^{\prime}-\cos 2 \vartheta\right) f \\
= & \left.\frac{\left(q^{\prime}\right)^{s_{1}}\left(q^{\prime \prime}\right)^{s_{2}}}{Q^{m+1}} \tilde{f}\right|_{0} ^{\infty}-\int_{0}^{\infty} \frac{s_{1}\left(q^{\prime}\right)^{s_{1}-1}\left(q^{\prime \prime}\right)^{s_{2}+1}+\left(q^{\prime}\right)^{s_{1}} s_{2}\left(q^{\prime \prime}\right)^{s_{2}-1} q^{\prime \prime \prime}}{Q^{m+1}} \\
& \quad+\int_{0}^{\infty} \frac{(m+1)\left(q^{\prime}\right)^{s_{1}+1}\left(q^{\prime \prime}\right)^{s_{2}}}{Q^{m+2}} \tilde{f}-\int_{0}^{\infty} \frac{\left(q^{\prime}\right)^{s_{1}}\left(q^{\prime \prime}\right)^{s_{2}}}{Q^{m+1}} f \cos 2 \vartheta
\end{aligned}
$$

where $2 n \tilde{f}$ is either $-\cos 2 n \vartheta$ or $\sin 2 n \vartheta$. Because of (4.5), the integrated term here is the boundary term in (ii). If $f=\cos 2 \vartheta$ in the last integral, we write $f \cos 2 \vartheta=\frac{1}{2}(1+\cos 4 \vartheta)$ to produce the integral term in (iii) together with an integral of the type $\left(m+1 ; s_{1}, s_{2}, 0\right)$. Otherwise, $f \cos 2 \vartheta$ produces either one or two integrals of this type. This completes the proof of the lemma.

Proof of theorem 4.1. Starting from the original integral

$$
\int_{0}^{\infty} \sin 2 \vartheta
$$


Table 1. Types of integrals arising in the proof of theorem 4.1

\begin{tabular}{ll}
\hline integral type & generates types \\
\hline$(0 ; 0,0,0)$ & $(1 ; 0,0,0),(2 ; 1,0,0)$ \\
$(1 ; 0,0,0)$ & $(2 ; 0,0,0),(3 ; 1,0,0)$ \\
$(2 ; 0,0,0)$ & $(3 ; 0,0,0),(4 ; 1,0,0)^{\mathrm{E}}$ \\
$(2 ; 1,0,0)$ & $(3 ; 1,0,0),(3 ; 0,1,0),(4 ; 2,0,0)$ \\
$(3 ; 0,0,0)$ & $(4 ; 0,0,0),(5 ; 1,0,0)^{\mathrm{E}}$ \\
$(3 ; 1,0,0)$ & $(4 ; 1,0,0)^{\mathrm{E}},(4 ; 0,1,0)^{\mathrm{E}, \mathrm{P}},(5 ; 2,0,0)^{\mathrm{E}, \mathrm{P}}$ \\
$(3 ; 0,1,0)$ & $(4 ; 0,1,0)^{\mathrm{E}, \mathrm{P}},(4 ; 0,0,1)^{\mathrm{E}, \mathrm{P}},(5 ; 1,1,0)^{\mathrm{E}, \mathrm{P}}$ \\
$(4 ; 0,0,0)$ & $(5 ; 0,0,0),(6 ; 1,0,0)^{\mathrm{E}}$ \\
$(4 ; 1,0,0)$ & $(5 ; 1,0,0)^{\mathrm{E}},(5 ; 0,1,0)^{\mathrm{E}, \mathrm{P}},(6 ; 2,0,0)^{\mathrm{E}, \mathrm{P}}$ \\
$(4 ; 2,0,0)$ & $(5 ; 2,0,0)^{\mathrm{E}, \mathrm{P}},(5 ; 1,1,0)^{\mathrm{E}, \mathrm{P}},(6 ; 3,0,0)^{\mathrm{E}, \mathrm{P}}$ \\
$(5 ; 0,0,0)$ & $(6 ; 0,0,0),(7 ; 1,0,0)^{\mathrm{E}}$ \\
$(5 ; 1,0,0)^{\mathrm{E}}$ & $(6 ; 1,0,0)^{\mathrm{E}},(6 ; 0,1,0)^{\mathrm{E}, \mathrm{P}},(7 ; 2,0,0)^{\mathrm{E}, \mathrm{P}}$ \\
\hline
\end{tabular}

which is of type $(0 ; 0,0,0)$, we obtain two integrals of types $(1 ; 0,0,0)$ and $(2 ; 1,0,0)$ as shown in (4.4). We then recursively apply lemma 4.2; the types of the resulting integrals in statement (i) of the lemma are summarized in table 1 . The superscript ' $\mathrm{P}$ ' or ' $\mathrm{E}$ ' indicates that integrals of this type satisfy the hypotheses of lemma 3.2 under condition $(\mathrm{P})$ or $(\mathrm{E})$, respectively, and there is therefore no further need to apply lemma 4.2 to them. The process indicated in table 1 stops fairly quickly except for integrals of the types

(1) $(m ; 0,0,0)$ under both conditions $(\mathrm{P})$ and $(\mathrm{E})$;

(2) $(m ; 1,0,0)$ under condition $(\mathrm{P})$.

In case (1) we have to continue until we reach a value of $m>2+2 / a$ by (3.7). In case (2), we need $m>3+1 / a$ by (3.4). (All integrals of other types which are produced along the way with $m \geqslant 6$ directly satisfy lemma 3.2.)

We now turn to the boundary terms in statement (ii) of lemma 4.2. Apart from the one given explicitly in (4.4), these terms are all const. $/ Q^{m+1}(0, \mu)$ with $m \geqslant 1$. The $\mu$-derivative of these terms thus contributes $O\left(\mu^{-3}\right)=o\left(\mu^{-2}\right)$ to (4.2).

Turning finally to the integral terms in statement (iii) of lemma 4.2 , we note that such a term first arises because of the $\cos 2 \vartheta$ in an integrand in (4.4) and, explicitly, this term is

$$
-\frac{1}{4} \int_{0}^{\infty} \frac{q^{\prime}}{Q^{3}}=\frac{1}{8 Q^{2}(0, \mu)} .
$$

Other similar terms which arise later in the process have a higher power of $Q$ and are negligible in comparison as $\mu \rightarrow \infty$. Thus, after the $\mu$-differentiation, these terms again contribute only $O\left(\mu^{-3}\right)$ to $(4.2)$.

It remains to check that (4.5) is satisfied for the relevant terms. These terms are multiples of $q^{\prime} / Q^{m}$ with $m \geqslant 3, q^{\prime \prime} / Q^{4}$ and $\left(q^{\prime}\right)^{2} / Q^{5}$, and (4.5) is clearly satisfied either directly from conditions $(\mathrm{P})$ and $(\mathrm{E})$ or from (3.2).

Altogether, then, the dominant term in (4.4) for large $\mu$ is $\frac{1}{2} \mu^{-1} \cos 2 \alpha$ and, as we have shown, it continues to provide the dominant term $-\frac{1}{2} \mu^{-2} \cos 2 \alpha$ after the 
Table 2. Types of integrals arising in addition if $o\left(\mu^{-3}\right)$ is required

\begin{tabular}{cl}
\hline integral type & generates types \\
\hline$(4 ; 1,0,0)$ & $(5 ; 1,0,0)^{\mathrm{E}},(5 ; 0,1,0)^{\mathrm{E}, \mathrm{P}},(6 ; 2,0,0)^{\mathrm{E}, \mathrm{P}}$ \\
$(4 ; 0,1,0)$ & $(5 ; 0,1,0)^{\mathrm{E}, \mathrm{P}},(5 ; 0,0,1)^{\mathrm{E}, \mathrm{P}},(6 ; 1,1,0)^{\mathrm{E}, \mathrm{P}}$ \\
$(4 ; 0,0,1)^{\mathrm{P}}$ & $(5 ; 0,0,1)^{\mathrm{E}, \mathrm{P}},(5 ; 0,0,0,1)^{\mathrm{E}, \mathrm{P}},(6 ; 1,0,1)^{\mathrm{E}, \mathrm{P}}$ \\
$(5 ; 2,0,0)$ & $(6 ; 2,0,0)^{\mathrm{E}, \mathrm{P}},(6 ; 1,1,0)^{\mathrm{E}, \mathrm{P}},(7 ; 3,0,0)^{\mathrm{E}, \mathrm{P}}$ \\
$(5 ; 1,1,0)^{\mathrm{P}}$ & $(6 ; 1,1,0)^{\mathrm{E}, \mathrm{P}},(6 ; 0,2,0)^{\mathrm{E}, \mathrm{P}},(6 ; 1,0,1)^{\mathrm{E}, \mathrm{P}},(7 ; 2,1,0)^{\mathrm{E}, \mathrm{P}}$ \\
$(6 ; 3,0,0)^{\mathrm{P}}$ & $(7 ; 3,0,0)^{\mathrm{E}, \mathrm{P}},(7 ; 2,1,0)^{\mathrm{E}, \mathrm{P}},(8 ; 4,0,0)^{\mathrm{E}, \mathrm{P}}$ \\
\hline
\end{tabular}

$\mu$-differentiation. Now (4.2) follows from (4.3). This concludes the proof of theorem 4.1 .

\section{Concluding remarks}

\subsection{The case $\cos 2 \alpha=0$}

If $\cos 2 \alpha=0$, the boundary term in (4.4) vanishes and, to obtain the asymptotic formula corresponding to (4.2), we need to exhibit the new dominant terms which arise from the integrals of types $(2 ; 1,0,0)$ and $(1 ; 0,0,0)$ in $(4.4)$. For the first integral, we have the term $\frac{1}{8} Q^{-2}(0, \mu)$ as explained in (4.6). For the second integral, integration by parts produces the boundary term

$$
-\frac{1}{8}(\cos 4 \alpha) Q^{-2}(0, \mu)=\frac{1}{8} Q^{-2}(0, \mu)
$$

since $\cos 2 \alpha=0$. Thus, we expect the new dominant term in (4.3) to be

$$
-\frac{1}{2} \frac{\partial}{\partial \mu} Q(0, \mu)^{-2}=\mu^{-3}(1+o(1)) \quad(\mu \rightarrow \infty) .
$$

This can indeed be proved by the method used to prove theorem 4.1, and we are now looking for $o\left(\mu^{-3}\right)$ estimates in place of the previous $o\left(\mu^{-2}\right)$ estimates.

Under condition $(\mathrm{P})$, we observe that in lemma 3.2(a) we can conclude that $\mathrm{d} I(\mu) / \mathrm{d} \mu=o\left(\mu^{-3}\right)$ if we have $m>3$ and

$$
m-\sigma+(\tau-2) / a>3
$$

in place of (3.4). The integrals of types $(4 ; 0,1,0)$ and $(5 ; 2,0,0)$ in table 1 do not satisfy this stronger condition, and so we must apply lemma 4.2 to them, resulting in integrals all of which fulfil (5.1) (see table 2). The additional boundary terms are multiples of $q^{\prime \prime} / Q^{5}$ and $\left(q^{\prime}\right)^{2} / Q^{6}$, which clearly vanish at infinity and have $\mu$-derivative $O\left(\mu^{-6}\right)$ at $x=0$.

In the case of condition (E), however, a slight complication ensues from the fact that the stronger condition for $o\left(\mu^{-3}\right)$ behaviour corresponding to (3.6) is

$$
m-\sigma>3+\delta
$$

and this condition now excludes the integral of type $(4 ; 0,0,1)$. In order to apply integration by parts to this integral, we require the existence of a fourth derivative of $q$. We therefore extend definition 3.1 and lemmas 3.2 and 4.2 to include the 
fourth derivative $q^{(4)}$, and we also allow $k=4$ in condition (E). We then note that, corresponding to (3.2), we now also have $x q^{\prime \prime \prime}(x) / q^{2}(x)=o(1)(x \rightarrow \infty)$ and, in particular, $q^{\prime \prime \prime}(x) / q^{5}(x)=o(1)(x \rightarrow \infty)$.

Then we can continue the process of recursively applying lemma 4.2 until all integrals satisfy (5.2), where now $\sigma=\sum_{j=1}^{4} s_{j}$ (see table 2). The boundary terms which appear are multiples of $q^{\prime} / Q^{5}, q^{\prime \prime} / Q^{5}, q^{\prime \prime \prime} / Q^{5},\left(q^{\prime}\right)^{2} / Q^{6}, q^{\prime} q^{\prime \prime} / Q^{6}$ and $\left(q^{\prime}\right)^{3} / Q^{7}$, and all have the required behaviour. Thus, we have established the following result.

THEOREM 5.1. Let $q$ be a potential satisfying (1.4) and (1.6); furthermore, assume that either

(a) $q$ is twice continuously differentiable and $q^{\prime \prime} \in A C_{\mathrm{loc}}(0, \infty)$ and condition $(P)$ holds, or

(b) $q$ is thrice continuously differentiable and $q^{\prime \prime \prime} \in A C_{\mathrm{loc}}(0, \infty)$ and condition (E) holds with $k \in\{1,2,3,4\}$.

Suppose further that $\cos 2 \alpha=0$. Then

$$
\pi \varrho_{\alpha}^{\prime \prime}(\mu)=\mu^{-3}(1+o(1)) \quad(\mu \rightarrow \infty) .
$$

In particular, there is an interval $(M, \infty)$ which does not contain any points of spectral concentration.

\subsection{Piecewise smooth $q$}

Suppose that $q$ is continuous in $(0, \infty)$ but that there are a finite number of points $x_{i}(i \in\{1,2, \ldots, n\})$, where $q$ fails to be differentiable. We still assume that, in each interval $\left(x_{i-1}, x_{i}\right)$ (with $\left.x_{0}=0\right), q$ is three times differentiable and satisfies condition $(\mathrm{P})$ or $(\mathrm{E})$. Then integration by parts in (4.4) produces an additional term

$$
\frac{1}{4} \sum_{i=1}^{n}\left[\frac{q^{\prime}}{Q^{3}} \sin 2 \vartheta\right](i)
$$

on the right-hand side, where $[\cdot](i)$ denotes the saltus at $x_{i}$. The subsequent integrations produce similar additional terms of order $O\left(\mu^{-4}\right)$.

After taking the $\mu$-derivative and using theorem 2.2, the effect of (5.4) is to introduce a generally indeterminate $O\left(\mu^{-3}\right)$ term into the right-hand side of (4.2), meaning that (4.2) continues to be valid. In the case of theorem 5.1, we would also need $q^{\prime}$ to be continuous in $(0, \infty)$ to make (5.4) vanish; then (5.3) continues to hold.

\section{Acknowledgments}

This work has received financial support from the EPSRC Spectral Theory Network.

\section{References}

1 B. M. Brown, M. S. P. Eastham and D. K. R. McCormack. Spectral concentration and rapidly decaying potentials. J. Computat. Appl. Math. 81 (1997), 333-348. 
2 B. M. Brown, M. S. P. Eastham and D. K. R. McCormack. Spectral concentration and perturbed discrete spectra. J. Computat. Appl. Math. 86 (1997), 415-425.

3 B. M. Brown, M. S. P. Eastham and D. K. R. McCormack. Absolute continuity and spectral concentration for slowly decaying potentials. J. Computat. Appl. Math. 94 (1998), 181-197.

4 E. A. Coddington and N. Levinson. Theory of ordinary differential equations (McGrawHill, 1955).

5 M. S. P. Eastham. Conditions for the spectrum in eigenfunction theory to consist of $(-\infty, \infty)$. Q. J. Math. 18 (1967), 147-153.

6 M. S. P. Eastham. The asymptotic solution of linear differential systems (Oxford: Clarendon, 1988).

7 M. S. P. Eastham. The asymptotic form of the spectral function in Sturm-Liouville problems with a large potential like $-x^{c}(c \leqslant 2)$. Proc. R. Soc. Edinb. A 128 (1998), 37-45.

8 M. S. P. Eastham. On the location of spectral concentration for Sturm-Liouville problems with rapidly decaying potential. Mathematika 45 (1998), 25-36.

9 M. S. P. Eastham. On the location of spectral concentration for perturbed discrete spectra. Mathematika 46 (1999), 145-154.

10 A. Erdélyi. Note on a paper by Titchmarsh. Q. J. Math. 14 (1963), 147-152.

11 D. J. Gilbert and B. J. Harris. Bounds for the points of spectral concentration of SturmLiouville problems. Mathematika 47 (2000), 327-337.

12 I. M. Glazman. Direct methods of qualitative spectral analysis of singular differential operators (Jerusalem: Israel Program for Scientific Translations, 1965).

13 S. G. Halvorsen. Counterexamples in the spectral theory of singular Sturm-Liouville operators. Ordinary and partial differential equations (ed. B. D. Sleeman and I. M. Michael). Springer Lecture Notes in Mathematics 415 (1974), 373-382.

14 P. Hartman. On the essential spectra of ordinary differential operators. Am. J. Math. 76 (1954), 831-838.

15 D. B. Hinton. Continuous spectra of second-order differential operators. Pac. J. Math. 33 (1970), 641-643.

16 B. M. Levitan and I. S. Sargsjan. Introduction to spectral theory: selfadjoint ordinary differential operators. AMS Translations, vol. 39 (Providence, RI: American Mathematical Society, 1975).

17 J. B. McLeod. On the continuous spectra of large negative potentials. Q. J. Math. 16 (1965), 233-240.

18 M. E. Rose and R. R. Newton. Properties of Dirac wave functions in a central field. Phys. Rev. 82 (1951), 470-477.

19 K. M. Schmidt. Absolutely continuous spectrum of Dirac systems with potentials infinite at infinity. Math. Proc. Camb. Phil. Soc. 122 (1997), 377-384.

20 K. M. Schmidt. A remark on the essential spectrum of Dirac systems. Bull. Lond. Math. Soc. 32 (2000), 63-70.

21 K. M. Schmidt and O. Yamada. Spherically symmetric operators with variable mass and potentials infinite at infinity. Publ. RIMS Kyoto 34 (1998), 211-227.

22 G. Stolz. Bounded solutions and absolute continuity of Sturm-Liouville operators. J. Math. Analysis Applic. 169 (1992), 210-228.

23 E. C. Titchmarsh. Eigenfunction expansions, part 1 (Oxford: Clarendon, 1946).

24 E. C. Titchmarsh. Eigenfunction expansions, part 1, 2nd edn (Oxford: Clarendon, 1962).

25 J. Walter. Absolute continuity of the essential spectrum of $-\mathrm{d}^{2} / \mathrm{d} t^{2}+q(t)$ without monotony of q. Math. Z. 129 (1972), 83-94.

(Issued 16 August 2005) 